\title{
Advances in the Chemopreventive Targeting of Oral Carcinogenesis
}

\author{
David J. Wisniewski ${ }^{1}$ Tao Ma ${ }^{1}$ - Abraham Schneider ${ }^{1,2}$
}

Published online: 2 April 2015

(C) Springer International Publishing AG 2015

\begin{abstract}
Control of oral cancer associated with the frequent exposure to traditional risk factors such as tobacco, betel quid, and alcohol, together with the recent rise in human papillomavirus-related oropharyngeal cancer is a major public health concern worldwide. Late diagnosis and "field cancerization," where multifocal malignant lesions compromise standard surgical treatments and chemoradiotherapy are among the most critical challenges faced by clinical oncologists. In addition to improving methods of early diagnosis and periodic monitoring, halting tumor progression through longterm chemoprevention of at risk premalignant lesions with well-tolerated drugs or natural products remains an attractive strategy. This review discusses recent progress and challenges on the chemopreventive targeting of oral cancer, especially as it relates to the genomic and molecular characterization of oncogenic drivers underlying tumor development and progression. These emerging data may ultimately serve as the basis for novel early and predictable interventions to significantly reduce morbidity and mortality associated with oral cancer.
\end{abstract}

Keywords Chemoprevention · Oral cancer · HNSCC · OSCC $\cdot$ EGFR $\cdot$ mTOR $\cdot$ Metformin $\cdot$ Review

This article is part of the Topical Collection on Oral Cancer

Abraham Schneider

aschneider@umaryland.edu

1 Department of Oncology and Diagnostic Sciences, School of Dentistry, University of Maryland, 650 W. Baltimore St., Room 7265, Baltimore, MD 21201, USA

2 Greenebaum Cancer Center, Program in Oncology, School of Medicine, University of Maryland, Baltimore, MD 21201, USA

\section{Introduction}

Head and neck squamous cell carcinoma (HNSCC), largely affecting the oral cavity and pharynx, is the sixth most common cancer in the world with approximately 500,000 new cases every year. Recent estimates indicate that in the United States more than 42,000 new cases of oral cancer, mostly oral squamous cell carcinomas (OSCC), were diagnosed in 2014. During the same year, approximately 8400 OSCC patients died as a consequence of complications from the disease $[1,2]$. Main risk factors for OSCC development in western countries include heavy tobacco use combined with excessive alcohol consumption, whereas in south and southeast Asia, OSCC is most commonly associated with betel and areca nut chewing $[3,4]$. In spite of an overall decline in tobacco consumption, the recent rise in head and neck cancer cases linked to oncogenic subtypes of human papillomaviruses (HPV) is becoming a growing public health concern. In contrast to OSCC, HPVassociated SCC develops mainly in the oropharynx, affects younger individuals, and demonstrates unique underlying molecular characteristics that lead to improved disease-specific patient survival $[5,6]$. Despite important advances in chemoradiotherapy and surgical approaches, the 5-year OSCC patient survival rate has not considerably changed over the last four decades ( $<40-50 \%)$, in part due to late diagnosis, multifocal distribution of primary malignant lesions, and tumor recurrence associated with treatment resistance of advanced cancer. The recent identification of highly prevalent oncogenic signatures underlying OSCC pathogenesis is providing the foundation not only to develop novel molecularly targeted strategies to control established OSCCs but also to foster more selective chemopreventive measures to halt the progression of oral precancerous lesions. The main objective of this review is to convey the most recent developments and challenges in the field of chemoprevention of oral cancer, in particular as it 
relates to the emerging characterization of the landscape of genomic, molecular, and cellular alterations underlying OSCC carcinogenesis.

\section{Definition of Chemoprevention}

Exposure to a variety of carcinogens as a result of heavy tobacco smoking and/or betel quid chewing together with alcohol consumption in different regions of the world remains the main risk factor for OSCC development. This makes OSCC one of the most preventable malignancies. The term primary cancer prevention denotes either avoiding or quitting these poor habits through educational and medical interventions. In fact, lack of exposure to carcinogens contained in these products would significantly reduce OSCC burden worldwide [7]. Secondary prevention is when at-risk populations are screened for early detection of disease or when neoplastic transformation of a potentially malignant lesion into a cancerous tumor is halted through chemopreventive measures. Tertiary prevention occurs when there is early detection of second primary malignant tumors following treatment of the original cancer [8]. Based upon the model of multistep carcinogenesis, secondary chemoprevention of oral cancer has emerged as an important type of intervention where a variety of drugs and natural products are used to interfere with the carcinogenic process and eventually deter disease progression.

\section{OSCC Pathogenesis}

As the lining of normal oral mucosa becomes continuously exposed to a variety of carcinogens, keratinocytes may be subjected to multiple genetic and epigenetic aberrations leading to cancer development. A process referred to as multistep carcinogenesis has been traditionally used to define OSCC development and progression as there have been observations of similar genetic alterations in both oral dysplastic, potentially malignant lesions, and fully established OSCCs $[9,10]$.

Clinically, oral leukoplakia is the most common potentially malignant oral lesion associated with OSCC development. This term indicates a white patch on the oral mucosa that cannot be scraped off and cannot be clinically classified as any other condition. It is found most commonly in smokers, but it can also appear in individuals with a non-smoking history. Oral leukoplakias may remain unchanged over time, regress spontaneously or by quitting tobacco use, or eventually transform into an invasive OSCC. To this end, later malignant transformation occurs in approximately 3 to $5 \%$ of benign leukoplakias [11]. Among the most worrying clinical signs are ulceration and erythematous foci (i.e., speckled erythroleukoplakia). However, about $5 \%$ of oral leukoplakias are already malignant at initial diagnosis. Therefore, it is of utmost importance to determine which lesional biomarkers together with other clinicopathological features will predict neoplastic transformation. Collectively, this may lead to the implementation of more selective, targeted chemopreventive strategies to interfere with commonly found oncogenic drivers of OSCC carcinogenesis $[8,12,13 \bullet \bullet]$.

Most likely, the identification of patients with at-risk, potentially malignant oral leukoplakias, where long-term chemopreventive treatment might be implemented and periodically monitored would result in better overall outcomes including improved quality of life and higher patient survival rates [14]. Unfortunately, it is currently difficult to determine the risk of malignant transformation as more information needs to be gained from a lesional biopsy. For instance, a 5 -year followup of patients who had been previously diagnosed with an oral leukoplakia showing high-grade dysplasia resulted in a higher OSCC incidence than those patients with low-grade dysplastic changes [15]. Overall, patients with leukoplakias with lowgrade dysplasia had a 5 -year OSCC free survival rate of $90.5 \%$ compared to only $59 \%$ in patients with high-grade dysplastic lesions. Some of the risk factors associated with this progression were age, lesions on the lateral/ventral tongue as well as heterogenous lesions such as erythroleukoplakias. It seems reasonable to suggest that the histopathological grading of dysplasias in combination with genetic and molecular biomarkers may prove useful for determining individualized biomarkers of neoplastic transformation. To this end, investigating the genomic and molecular landscape of OSCC becomes a priority research endeavor $[13 \bullet \bullet, 16,17]$.

\section{The Emerging HNSCC/OSCC Oncogenome}

Deregulated signal transduction pathways play a dominant role in supporting the malignant behavior of cancer cells by altering the homeostatic control of key cellular processes such as cell cycle progression, cell metabolism, survival, motility, and angiogenesis. In recent years, cancer biologists have been actively engaged in dissecting the complex nature of major oncogenic networks to primarily understand their aberrant role within cancer cells and the surrounding tumor microenvironment. Ideally, more actionable targets should become available to control cancer development and progression, including OSCCs, as more genomic alterations and deregulated molecular pathways are systematically identified $[18 \bullet, 19]$. In this regard, the recent characterization of the HNSCC/OSCC oncogenome through comprehensive genomic analysis in human-derived tumors and cell lines is of great value to identify novel mutations, changes in gene expression, epigenetic gene signatures, and aberrant molecular signaling pathways $[13 \bullet, 20 \bullet, 21 \bullet \bullet$. Indeed, significantly more somatic gene mutations have been identified in HNSCC/OSCCs than 
previously reported. An important translationally relevant challenge in the field, however, is determining which mutations are key "driver" mutations and which are "passenger" mutations that minimally impact tumor progression and treat-

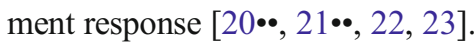

Through the systematic identification of highly prevalent genomic and molecular signatures underlying HNSCC/OSCC pathogenesis, recently gathered information indicates that a large number of tumors become dependent on the uncontrolled activation of multiple mitogenic signaling pathways; however, those closely related to the epidermal growth factor receptor (EGFR) and the phosphatidylinositol 3'-kinase (PI3K)-Akt-mammalian target of rapamycin (mTOR) signaling networks are among the most critically involved in tumor cell growth and survival [13••, 21••, 24, 25].

By using fluorescent in situ hybridization (FISH), EGFR and $C C N D 1$ gene copy number alterations have been recently reported in oral premalignant lesions with high-grade dysplasias. Of note, $C C N D 1$ encodes the protein cyclin D1, an important component of the cell cycle machinery [26]. To this end, high gene gains in EGFR and CCND1 were associated with an elevated risk of tumor progression [17]. Another recent study in $151 \mathrm{HNSCC/OSCC}$ tumors revealed the PI3K signaling network as the most frequently mutated oncogenic pathway $(30.5 \%)[21 \cdot \bullet]$. Within the PI3K pathway, the $P I K 3 C A$ gene is largely the most frequently mutated in HNSCC/OSCC tumors and cell lines [13••, 21••]. However, other members in this pathway also appear to be affected. Loss of the tumor suppressor PTEN, which negatively impacts the PI3K-driven mitogenic signals, is found in approximately $30 \%$ of HNSCC/OSCCs. The widespread activation of the mTOR pathway in hundreds of human HNSCC/OSCC tumor specimens has also been reported [27]. High levels of phosphorylated ribosomal S6 protein (pS6), a well-known downstream effector in the mTOR pathway and a common surrogate marker for mTOR activity, have been identified in more than $90 \%$ of HNSCC/OSCC cases. Increased pS6 levels are observed not only in HNSCC/OSCC but also in advanced oral premalignant lesions where $\mathrm{pS} 6$ moderate to strong immunoreactivity has been noted $[28,29]$. Since the majority of HNSCC/OSCCs are driven through these signaling pathways, targeting of potentially malignant oral lesions relying on these oncogenic networks could be exploited with chemopreventive strategies $[21 \bullet \bullet, 30]$.

\section{Chemoprevention of Oral Carcinogenesis}

The majority of potential chemopreventive drugs and natural compounds may generally be perceived as safe and non-toxic, but this notion does not seem to be accurate. Considering the potential long-term use of a chemopreventive drug or natural compound, key issues playing a critical role in the clinical decision-making process include determining the appropriate concentration, potency, and toxicity of the active compound as well as elucidating the underlying mechanism of antineoplastic action. Following on the emerging characterization of the HNSCC/OSCC mutational landscape, chemopreventive drug discovery should also be based on the identification of genomic and molecular markers that frequently correlate with tumor progression. This may provide the basis for implementing optimal long-term targeting approaches with cost-effective, non-toxic compounds [31]. With a particular emphasis on the EGFR and PI3K/mTOR signaling pathways, we will discuss in more detail their relative contribution to head and neck/oral carcinogenesis and report on emerging preclinical and clinical evidence utilizing chemopreventive targeting to pharmacologically block their constant activation.

\section{Targeting EGFR Signaling}

A large number of targeted drugs have been developed, marketed, and used in the clinical setting to positively control tumor development and progression by improving treatment responses, patient quality of life, and overall survival. A clear example of a bench-to-bedside breakthrough in oncology took place with the introduction of small molecules and monoclonal antibodies aimed at blocking the activity of the ErbB family of transmembrane receptor tyrosine kinases, in particular, the ErbB1 or epidermal growth factor receptor (EGFR) [32, 33]. After more than 30 years of the initial report of a close genetic similarity between a viral oncogene encoding the verb-B transforming protein of avian erythroblastosis with a truncated active mutant of the human ERBB gene, it is apparent that the malignant phenotype of cancer cells can be initiated and maintained by intracellular signals transmitted through dysfunctional EGFR [34]. Cancers of the lung, colon, pancreas, brain, and the head and neck often present with EGFR somatic genetic alterations that negatively affect intracellular signaling cascades that are linked to normal EGFR function. Many intervening signaling factors downstream of the EGFR are critical for regulating cell growth, proliferation, survival, and motility in different cell types of epithelial and mesenchymal origin [32].

In the case of lung cancer and non-small cell lung carcinomas (NSCLC) in particular, clinical studies support the notion that positive responses to EGFR targeted agents are better predicted based on the underlying mutation(s) affecting the EGFR gene rather than on traditional clinicopathological characteristics. Noteworthy, a sustained clinical benefit to tyrosine kinase inhibitors (TKI) like gefitinib or erlotinib, used as single agents, has proven to be more effective in a subset of NSCLC patients with tumors containing EGFR activating mutations [35-38]. Resistance to TKI, on the contrary, occurs more frequently in NSCLC patients where no EGFR mutations are present $[39,40]$. 
These groundbreaking findings have provided a better understanding of how treatment sensitivity to molecularly based cancer therapies may be directly associated with the presence of unique mutations in the gene encoding a specific targeted protein. These studies not only underscore the impact the tumor genetic framework may exert on therapeutic decisionmaking but also offer unprecedented opportunities for screening the genotype of individual tumors through molecular and genomic profiling approaches to predict clinical responses and improve treatment outcomes [29, 38, 41-43].

Nonetheless, questions still remain in regard to the lack of overall positive responses to EGFR targeted drugs in a considerable number of patients with different cancer types when administered as a single agent where the wild-type EGFR gene is highly expressed but not mutated. Indeed, this scenario has been clearly exemplified by the relatively limited clinical efficacy following the use of the anti-EGFR monoclonal antibody cetuximab in patients with HNSCC/OSCCs, where the EGFR and some of its ligands are overexpressed in 50-100\% of cases [44, 45]. In addition to the presence of a constitutive active truncated mutant form, the EGFR variant III, in approximately $40 \%$ of HNSCC/OSCC cases and its association with cetuximab resistance [46-48], a large segment of the HNSCC/ OSCC population harbors increased copy numbers of the wild-type EGFR in their premalignant and malignant cells. With more than 12 copies per cell described in some HNSC C/OSCC tumors [49], EGFR amplification is the major mechanism for EGFR overexpression in head and neck/oral cancer and is consistently associated with poor clinical outcomes [17, 49-53]. Furthermore, the 5-year survival for HNSCC/OSCC patients with increased EGFR copy number is only $9 \%$, in comparison to $71 \%$ in those patients with normal copy numbers [49]. It is also apparent that a large number of HNSCC/ OSCC tumors become dependent on the uncontrolled activation of multiple signaling pathways closely related to the EGFR signaling network for their aberrant growth and survival [25]. This provides the rationale for exploiting multitarget treatment modalities that combine two or more agents to specifically hit intervening signaling factors within the same pathway or preventing the crosstalk of different pathways.

Among several important signaling effectors that are activated in HNSCC/OSCC downstream of the EGFR is the signal transducer and activator of transcription (STAT) pathway. Immune responses, stem cell function, cell growth, differentiation, and organogenesis are among the biological processes governed by the activity of genes targeted by the STAT family of transcription factors (STAT1, 2, 3, 4, 5a, 5b, and 6) [54]. Elevated STAT3 expression and phosphorylation levels have been often found in HNSCC/OSCC tumors and their adjacent normal epithelia suggesting an early role in head and neck/oral carcinogenesis [55]. Moreover, constitutively active phosphorylated STAT3 is closely linked to the EGFR signaling cascade and correlates with HNSCC/OSCC metastatic spread and poor prognosis $[55,56]$. The role of STAT- 3 in head and neck/oral carcinogenesis is significant since blocking STAT3 activity results in tumor growth inhibition both in vitro and in vivo $[57,58]$. In this regard, Leeman-Neill et al. recently used a mouse model of oral cancer induced by the administration of the DNA adduct-forming carcinogen 4nitroquinoline-1-oxide (4-NQO), which mimics human multistage oral carcinogenesis, to study the chemopreventive activity of two different compounds targeting the EGFR-STAT3 signaling pathway [59]. Dietary guggulipid, an extract of a medicinal plant containing the STAT3 inhibitor guggulsterone, failed to protect against carcinogenesis; however, mice given an erlotinib-supplemented diet exhibited a $69 \%$ decrease in the incidence of oral premalignant and OSCC lesions compared with mice having the control diet. These preclinical results provided the basis for exploring the use of erlotinib in OSCC chemoprevention.

In fact, erlotinib is in phase III development for oral cancer prevention [60]. In a small open-label, clinical trial $(n=31$ patients), patients with recurrent $\mathrm{HNSCC} / \mathrm{OSCC}$ were treated with adjuvant $150 \mathrm{mg}$ /day erlotinib (Tarceva ${ }^{\mathbb{R}}$; Genentech Inc., San Francisco, CA) for 1 year following salvage surgery (tertiary chemoprevention). Primary outcome measures included tolerance to long-term erlotinib therapy, disease-free survival, and overall survival at 1 and 2 years. A total of eight patients concluded the protocol. Another eight patients withdrew before the conclusion of the study due to tumor recurrence; ten patients discontinued the study due to toxicities, including rash, diarrhea, and fatigue; three patients left due to other medical conditions; and two patients were discontinued because of noncompliance. Overall survival was $61 \%$ at 1 year and $56 \%$ at 2 years. Disease-free survival was $54 \%$ at 1 year and $45 \%$ at 2 years. Although, erlotinib showed a potential slight benefit in overall patient and diseasefree survival, the poor tolerance of the drug in the postoperative setting even at conventional dosing levels affected the completion of the 1-year study. Future studies with lower erlotinib doses may lead to minimal manageable toxicities and more promising therapeutic responses [61•].

Emerging evidence also demonstrates that EGFR TKI's (erlotinib) and selective inhibitors of the pro-inflammatory cyclooxygenase-2 (COX-2) pathway (celecoxib) can act synergistically to prevent tumorigenesis in $\mathrm{HNSCC} / \mathrm{OSCC}$ cell line-derived tumor xenografts grown in mice [62]. Cyclooxygenases catalyze the formation of the proinflammatory prostaglandins from arachidonic acid. Early studies determined that $\mathrm{COX}-2$, the inducible form of cyclooxygenases, was strongly upregulated in oral premalignant lesions and HNSCC/OSCCs, and that this response could also be triggered through EGFR signaling. High COX-2 levels may contribute to cancer development and progression by regulating apoptosis, immune surveillance, and angiogenesis $[63,64]$. In the recent Shin et al. study, the combined treatment 
of erlotinib and celecoxib inhibited tumor growth significantly and more potently than either drug alone. In particular, this treatment markedly affected the activity of the EGFR and mTOR signaling pathways. When this novel combination therapy was investigated in a phase I chemoprevention clinical trial, the overall pathologic response rate reached $71 \%$. Immunohistochemistry performed in tissue samples obtained from responding patients post treatment consistently demonstrated an inhibitory action on EGFR and mTOR pathways. Although a larger number of patients are needed to strongly support the results of this study, this drug combination may offer an attractive chemopreventive strategy by targeting the EGFR and mTOR pathways, which may ultimately serve as potential biomarkers to follow-up patient response $[62,65 \bullet$.

\section{The Antidiabetic Drug Metformin in Oral Carcinogenesis}

A recurring theme in cancer biology and therapy is that common drugs used to treat other chronic diseases, such as the COX-2 selective nonsteroidal anti-inflammatory drugs for osteoarthritic conditions, may also exert antitumorigenic activities. A similar scenario appears to be true with metformin, the most widely used oral medication for the control of type 2 diabetes mellitus worldwide. The biguanide metformin was originally isolated from guanidine, the active component of the French lilac Galega officinalis [66]. The role of antidiabetic drugs in cancer was initially attributed to recent retrospective population studies indicating that metformin treatment in diabetics is linked to better cancer outcomes when compared to other antihyperglycemic medications like insulin [67]. A large number of preclinical and clinical studies have been carried out since then demonstrating a positive effect of metformin in controlling the development and progression of several types of cancer [68, 69].

The underlying mechanism of antineoplastic action of metformin is most likely mediated via the AMP-activated protein kinase (AMPK) pathway, a conserved signaling network acting as a master sensing mechanism to intracellular bioenergetic stress and a known inhibitor of mTOR activity [69]. Furthermore, metformin negatively impacts tumor cell proliferation by downregulating mTOR signaling-mediated translation of a specific subset of cell cycle regulators [70]. As a mild inhibitor of mitochondrial respiration, metformin restricts mTOR activity by activating the AMPK pathway. Although AMPK is not a direct target of metformin, it becomes activated following metformin-induced decrease in intracellular energy status characterized by an increase in the cellular AMP/ ATP ratio [71-74].

Emerging evidence points to the mTOR signaling pathway as a highly prevalent overactivated molecular network underlying HPV-negative and HPV-positive head and neck carcinogenesis [30]. Significant chemopreventive effects have already been observed using clinically relevant doses of the
mTOR inhibitor rapamycin in the 4NQO-induced mouse model of oral carcinogenesis. In this setting, rapamycin administration significantly prevented the malignant conversion of oral precancerous lesions and induced the regression of advanced OSCCs [29]. However, the long-term use of an immunosuppressing drug like rapamycin might trigger potential unwanted side effects. To this end, the notion of incorporating metformin, a well-known drug with more than 50 years of clinical use, relatively inexpensive, safe, and well tolerated in the chemopreventive clinical management of oral cancer is promising.

Encouraging preclinical positive responses to metformin in oral carcinogenesis have been recently identified [75•]. VitaleCross et al. took advantage of the 4NQO-induced oral carcinogenesis mouse model to study the chemopreventive effects of metformin in the progression of oral premalignant lesions with variable degrees of epithelial dysplasia. Following 14 weeks of $4 \mathrm{NQO}$ administration in drinking water $(50 \mu \mathrm{g} /$ $\mathrm{mL}$ ), mice were randomly assigned to either daily intraperitoneal injections of metformin $(50 \mathrm{mg} / \mathrm{kg} / \mathrm{day})$ or sterile saline (control group). After 8 weeks of treatment, the number of progressing oral lesions, total tumor multiplicity, and OSCC multiplicity was significantly reduced in metformin-treated mice. Immunohistochemical analysis of tissue specimens revealed a clear inhibition of mTOR activity, as evidenced by reduced pS6 immunostaining, primarily in the basal/ suprabasal proliferating dysplastic epithelium. Of note, no changes in metabolic markers such as glucose, insulin, and insulin-like growth factor-1 (IGF-1) were evident in the immunocompetent, nonobese, and nondiabetic mice. Moreover, our group has also recently reported that members of the solute carrier 22A (SLC22A) family of organic cation transporters (OCT), in particular OCT-3, which facilitate the intracellular uptake and activity of metformin, a hydrophilic cationic compound, are highly expressed in human oral epithelial dysplasias and well-differentiated OSCCs [76]. Conversely, in poorly differentiated OSCC tumors, OCT-3 immunostaining is weak or absent. These findings position OCT-3 as a potential tumor biomarker and point to highly OCT-3-expressing oral premalignant lesions or early OSCC tumors as ideal target tissues for metformin use. Collectively, these results should serve as the basis for future clinical trials aimed at optimizing the potential use of metformin in the oral oncology setting.

\section{Natural Compounds as Chemopreventive Agents}

Patients with oral premalignant lesions, as well as patients who have a higher risk for developing primary or recurrent OSCC will be ultimately the ones benefiting the most from chemoprevention. Yet, since these interventions will be targeting otherwise healthy patients, a chemopreventive compound should require to be non-toxic, trigger manageable side 
effects, if any, and reassure long-term patient compliance. In recent years, different research groups have reported on the beneficial chemopreventive effects of various natural products including grape seed extract, freeze-dried berries, and green tea. In a preclinical study using the $4 \mathrm{NQO}$ oral carcinogenesis mouse model, Shrotriya et al. investigated the role of grape seed extract and resveratrol in the chemoprevention of tongue tumorigenesis. After 8 weeks of $4 \mathrm{NQO}$ consumption in drinking water, mice were given either a control diet or a diet containing $0.2 \%$ grape seed extract or $0.25 \%$ resveratrol for eight additional weeks, while still on $4 \mathrm{NQO}$. At the completion of the study (16 weeks), mice on both of the test diets had a moderate decrease in OSCC incidence but a significant preventive effect on the multiplicity and severity of 4NQOinduced premalignant and malignant lesions. Through immunohistochemical analyses, the authors show that in tongues from mice given grape seed extract or resveratrol supplementation, there was a decrease in tumor cell proliferation, increase in tumor cell apoptosis, and higher phosphorylated AMPK expression levels. Collectively, these promising findings point to the potential benefit that grape seed extract and resveratrol, possibly via AMPK signaling, might have on the chemoprevention of oral carcinogenesis [77].

In another preclinical study using a chemical carcinogenesis model in hamster cheek pouches, Warner et al. reported on the favorable topical effects of a freeze-dried black raspberry suspension on the progression of oral premalignant lesions. Following a 12-week study, the authors observed that in comparison to controls, the freeze-dried black raspberry-treated group had reduced tumor multiplicity $(\sim 4 \%)$, tumor incidence $(\sim 37 \%)$, and tumor cell proliferation rate $(\sim 7 \%)$ [78]. Likewise, the same research group studying oral carcinogenesis in a similar animal model demonstrated that lyophilized strawberries present in the diet may trigger positive effects on the chemoprevention of oral cancer. Following 12 weeks of treatment, histopathological analyses revealed a significant reduction in the number of potentially malignant oral dysplastic lesions and malignant tumors in animals given lyophilized strawberries [79]. Although these studies are definitely thought provoking, it still remains unclear which underlying molecular mechanisms are associated with the promising antineoplastic responses.

Green tea polyphenols are antioxidants with potential inhibitory actions on various oncogenic signaling pathways including receptor tyrosine kinases such as EGFR [8]. A recent meta-analysis study was conducted to assess whether tea consumption had any impact on the risk of oral cancer [80]. Whereas green tea consumption among Asian and Caucasian populations was associated with a lower risk of oral cancer, these effects were not associated with black tea. This suggests that green tea may have a protective effect on the development of oral cancer. Similar significant effects were observed in another study where green tea intake was associated significantly with a reduced risk of oral cancer in men, but not in women, especially in those smoking heavily [81].

\section{Nanotechnology in Cancer Chemoprevention}

While many bioactive products have proved to be effective in preclinical models of cancer chemoprevention, disappointment has often occurred when these compounds are used clinically. Compounds such as resveratrol, curcumin, thymoquinone, and epigallocatechin (EGCG) have shown potent anticancer activity in cell culture and preclinical models of cancer, but their hydrophobicity and poor water solubility have resulted in less than optimal absorption and bioavailability [82]. To overcome these limitations, the use of nanoparticles has been explored in order to improve drug delivery and reduce toxic side effects. Nanotechnology is the study of materials on atomic and molecular scales with arrangements in the nanometer size range $\left(10^{-9} \mathrm{~nm}\right)$. The type of nanoparticle depends mostly on the physicochemical properties of the drug to be loaded. For instance, liposomes (artificial phospholipid vesicles) are effectively loaded with water-soluble agents. In contrast, micelles or colloidal dispersions with a particle size between 5 and $100 \mathrm{~nm}$ are a better option for water-insoluble drugs like the ones mentioned above. Micelles made up of biodegradable polymers such as polylactic acid, poly (DL-lactide-co-glycolide acid) (PLGA), starch, and chitosan increase the solubility and bioavailability of poorly soluble pharmaceutical agents. Early developments in this field relative to chemoprevention coined the term "nanochemoprevention" [83]. To this end, encapsulated green tea polyphenol EGCG in polylactic acid-polyethylene glycol nanoparticles showed improved biological effectiveness with over a 10-fold dose advantage [83]. These early findings encouraged the production of other nanochemopreventive formulations including nanocurcumin $[84,85]$, nanoresveratrol [86-88], genistein nanoencapsulation [89], and nanoformulations of the chemotherapeutic agent paclitaxel $[90,91]$. All of these nanoformulations have demonstrated improved pharmacokinetic and pharmacodynamic properties. The role of nanochemoprevention in oral cancer has been largely unexplored. However, in one study using the 7,12dimethyl benz(a)anthracene (DMBA)-induced multistage oral carcinogenesis in hamsters, orally administered naringeninloaded nanoparticles (40-90 $\mathrm{nm}$ size range and $\sim 88 \%$ encapsulation efficiency) showed positive antitumoral responses compared to free naringenin [92]. Naringenin is a type of flavonoid commonly found in grapefruit that has been shown to have antioxidant and anti-inflammatory effects [93]. A1though in a very primitive state and with much research still needed in the future, these results may lead to 
novel formulations that could be potentially utilized in the nanochemoprevention of oral cancer.

\section{Conclusions}

The chemopreventive targeting of oral cancer development and progression continues to be an attractive area of basic and clinical research. There is no doubt that major strides have been made in recent years by deciphering the oncogenomic

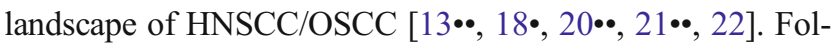
lowing on these promising findings, identifying common genomic and molecular signaling nodes and biomarkers of neoplastic transformation in oral premalignant lesions should also ultimately aid in the stratification and selection of at-risk patients. Moreover, this will eventually contribute to the development and clinical application of more predictable chemopreventive drugs $[8,14,94]$. However, this task offers many challenges, especially in a malignant disease where traditional risk factors such as tobacco use and betel quid chewing are still largely responsible for its occurrence. For instance, patients may be advised to quit their smoking habits, and over time, this may contribute to the regression of premalignant lesions. Yet, this positive behavioral step may mask any potential benefit of an administered chemopreventive drug. Further, the risk associated with malignant transformation is extremely variable and may not represent the drug effect. Therefore, to demonstrate the effectiveness of the drug, regression of any potentially premalignant lesion must be directly linked to the effects of the chemopreventive drug [31].

In this review, we have mainly focused our attention on recent studies reporting novel targeting $\mathrm{HNSCC} / \mathrm{OSCC}$ chemoprevention directed at interfering with the commonly deregulated oncogenic EGFR, PI3K, and mTOR pathways either by using selective drugs or common drugs marketed for other chronic conditions (i.e., celecoxib; metformin). Likewise, with the goal of reducing oral carcinogenesis, other novel chemopreventive strategies involving the systemic or topical delivery of natural products like green tea, grape seed extract, freeze-dried raspberries, and strawberries have been also used in high at-risk oral mucosa [77-80]. An important multidisciplinary area that deserves more attention is associated with the emerging developments in the field of nanochemoprevention. Hopefully, novel nanoformulations may eventually find a place in the chemopreventive setting of oral carcinogenesis.

\section{Compliance with Ethics Guidelines}

Conflict of Interest David J. Wisniewski, Tao Ma, and Abraham Schneider declare that they have no conflict of interest.

Human and Animal Rights and Informed Consent This article does not contain any studies with human or animal subjects performed by any of the authors.

\section{References}

Papers of particular interest, published recently, have been highlighted as:

- Of importance

- Of major importance

1. Campisi G, Giovannelli L. Controversies surrounding human papilloma virus infection, head \& neck vs oral cancer, implications for prophylaxis and treatment. Head Neck Oncol. 2009;1:8.

2. Siegel R, Ma J, Zou Z, Jemal A. Cancer statistics, 2014. CA Cancer J Clin. 2014;64(1):9-29.

3. Leemans CR, Braakhuis BJM, Brakenhoff RH. The molecular biology of head and neck cancer. Nat Rev Cancer. 2011;11(1):9-22.

4. Lee CH, Ko AM, Warnakulasuriya S, Ling TY, Sunarjo, Rajapakse PS, et al. Population burden of betel quid abuse and its relation to oral premalignant disorders in South, Southeast, and East Asia: an Asian Betel-quid Consortium Study. Am J Public Health. 2012;102(3):e17-24.

5. van Monsjou HS, Balm AJ, van den Brekel MM, Wreesmann VB. Oropharyngeal squamous cell carcinoma: a unique disease on the rise? Oral Oncol. 2010;46(11):780-5.

6. Panwar A, Batra R, Lydiatt WM, Ganti AK. Human papilloma virus positive oropharyngeal squamous cell carcinoma: a growing epidemic. Cancer Treat Rev. 2014;40(2):215-9.

7. Blackburn EH. Highlighting the science of cancer prevention. Cancer Prev Res (Phila). 2010;3(4):393.

8. Foy J-P, Bertolus C, William WN, Saintigny P. Oral premalignancy: the roles of early detection and chemoprevention. Otolaryngol Clin N Am. 2013;46(4):579-97.

9. Partridge M, Emilion G, Pateromichelakis S, Phillips E, Langdon J. Field cancerisation of the oral cavity: comparison of the spectrum of molecular alterations in cases presenting with both dysplastic and malignant lesions. Oral Oncol. 1997;33(5):332-7.

10. Califano J, van der Riet P, Westra W, Nawroz H, Clayman G, Piantadosi S, et al. Genetic progression model for head and neck cancer: implications for field cancerization. Cancer Res. 1996;56(11):2488-92.

11. Regezi JA, Sciubba JJ, Jordan RCK. Oral pathology: clinical pathologic correlations. 5th ed. Saunders Elsevier: St. Louis; 2008.

12. Lee JJ, Hong WK, Hittelman WN, Mao L, Lotan R, Shin DM, et al. Predicting cancer development in oral leukoplakia: ten years of translational research. Clin Cancer Res. 2000;6(5):170210 .

13.• Martin D, Abba MC, Molinolo AA, Vitale-Cross L, Wang Z, Zaida $\mathrm{M}$, et al. The head and neck cancer cell oncogenome: a platform for the development of precision molecular therapies. Oncotarget. 2014;5(19):8906-23. An important study characterizing by full exome and transcriptome sequencing a large panel of HNSCCderived cells from different anatomical locations and HPV infection status. Results showed widespread activation of the PI3K-mTOR pathway.

14. Dionne KR, Warnakulasuriya S, Binti Zain R, Cheong SC. Potentially malignant disorders of the oral cavity: current practice and future directions in the clinic and laboratory. Int $\mathrm{J}$ Cancer. 2015;136(3):503-15.

15. Liu W, Shi L-J, Wu L, Feng J-Q, Yang X, Li J, et al. Oral cancer development in patients with leukoplakia-clinicopathological factors affecting outcome. PLoS One. 2012;7(4):e34773.

16. Kujan O, Oliver RJ, Khattab A, Roberts SA, Thakker N, Sloan P. Evaluation of a new binary system of grading oral epithelial dysplasia for prediction of malignant transformation. Oral Oncol. 2006;42(10):987-93. 
17. Poh CF, Zhu Y, Chen E, Berean KW, Wu L, Zhang L, et al. Unique FISH patterns associated with cancer progression of oral dysplasia. J Dent Res. 2012;91(1):52-7.

18. Iglesias-Bartolome R, Martin D, Gutkind JS. Exploiting the head and neck cancer oncogenome: widespread PI3K-mTOR pathway alterations and novel molecular targets. Cancer Discov. 2013;3(7): $722-5$. An outstanding review summarizing the emerging contribution of the mutational landscape in HNSCC/OSCC.

19. Garraway LA, Lander ES. Lessons from the cancer genome. Cell. 2013;153(1):17-37.

20.• Pickering CR, Zhang J, Yoo SY, Bengtsson L, Moorthy S, Neskey $\mathrm{DM}$, et al. Integrative genomic characterization of oral squamous cell carcinoma identifies frequent somatic drivers. Cancer Discov. 2013;3(7):770-81. This study conducted a comprehensive genomic analysis of gene expression, copy number, point mutations and epigenetic changes in OSCC. Identified the Notch pathway highly defective in OSCC tumors. The authors found that $80 \%$ of patients carried at least one targetable genomic alteration.

21.• Lui VWY, Hedberg ML, Li H, Vangara BS, Pendleton K, Zeng Y, et al. Frequent mutation of the PI3K pathway in head and neck cancer defines predictive biomarkers. Cancer Discov. 2013;3(7): 761-9. This study analyzed whole-exome sequencing data from 151 HNSCC/OSCC tumors. Identified the PI3K pathway as the most frequently mutated oncogenic pathway in HNSCC/OSCC.

22. Agrawal N, Frederick MJ, Pickering CR, Bettegowda C, Chang K, $\mathrm{Li} \mathrm{RJ}$, et al. Exome sequencing of head and neck squamous cell carcinoma reveals inactivating mutations in NOTCH1. Science. 2011;333(6046):1154-7.

23. Stransky N, Egloff AM, Tward AD, Kostic AD, Cibulskis K, Sivachenko A, et al. The mutational landscape of head and neck squamous cell carcinoma. Science. 2011;333(6046):1157-60.

24. Freier K, Joos S, Flechtenmacher C, Devens F, Benner A, Bosch FX, et al. Tissue microarray analysis reveals site-specific prevalence of oncogene amplifications in head and neck squamous cell carcinoma. Cancer Res. 2003;63(6):1179-82.

25. Molinolo AA, Amornphimoltham P, Squarize CH, Castilho RM, Patel V, Gutkind JS. Dysregulated molecular networks in head and neck carcinogenesis. Oral Oncol. 2008.

26. Inaba T, Matsushime H, Valentine M, Roussel MF, Sherr CJ, Look AT. Genomic organization, chromosomal localization, and independent expression of human cyclin D genes. Genomics. 1992;13(3): 565-74.

27. Molinolo AA, Hewitt SM, Amornphimoltham P, Keelawat S, Rangdaeng S, Meneses García A, et al. Dissecting the Akt/ mammalian target of rapamycin signaling network: emerging results from the head and neck cancer tissue array initiative. Clin Cancer Res. 2007;13(17):4964-73.

28. Amornphimoltham P, Patel V, Sodhi A, Nikitakis NG, Sauk JJ, Sausville EA, et al. Mammalian target of rapamycin, a molecular target in squamous cell carcinomas of the head and neck. Cancer Res. 2005;65(21):9953-61.

29. Czerninski R, Amornphimoltham P, Patel V, Molinolo AA, Gutkind JS. Targeting mammalian target of rapamycin by rapamycin prevents tumor progression in an oral-specific chemical carcinogenesis model. Cancer Prev Res (Phila). 2009;2(1):27-36.

30. Molinolo AA, Marsh C, El Dinali M, Gangane N, Jennison K, Hewitt S, et al. mTOR as a molecular target in HPV-associated oral and cervical squamous carcinomas. Clin Cancer Res. 2012;18(9): 2558-68.

31. William WN, Papadimitrakopoulou VA. Optimizing biomarkers and endpoints in oral cancer chemoprevention trials. Cancer Prev Res (Phila). 2013;6(5):375-8.

32. Laurent-Puig P, Lievre A, Blons H. Mutations and response to epidermal growth factor receptor inhibitors. Clin Cancer Res. 2009;15(4):1133-9.
33. Shepard HM, Brdlik CM, Schreiber H. Signal integration: a framework for understanding the efficacy of therapeutics targeting the human EGFR family. J Clin Invest. 2008;118(11):3574-81.

34. Downward J, Yarden Y, Mayes E, Scrace G, Totty N, Stockwell P, et al. Close similarity of epidermal growth factor receptor and v-erbB oncogene protein sequences. Nature. 1984;307(5951):521-7.

35. Fukuoka M, Yano S, Giaccone G, Tamura T, Nakagawa K, Douillard JY, et al. Multi-institutional randomized phase II trial of gefitinib for previously treated patients with advanced non-smallcell lung cancer (The IDEAL 1 Trial) [corrected]. J Clin Oncol. 2003;21(12):2237-46.

36. Lynch TJ, Bell DW, Sordella R, Gurubhagavatula S, Okimoto RA, Brannigan BW, et al. Activating mutations in the epidermal growth factor receptor underlying responsiveness of non-small-cell lung cancer to gefitinib. N Engl J Med. 2004;350(21):2129-39.

37. Paez JG, Janne PA, Lee JC, Tracy S, Greulich H, Gabriel S, et al. EGFR mutations in lung cancer: correlation with clinical response to gefitinib therapy. Science. 2004;304(5676):1497-500.

38. Sequist LV, Martins RG, Spigel D, Grunberg SM, Spira A, Janne PA, et al. First-line gefitinib in patients with advanced non-smallcell lung cancer harboring somatic EGFR mutations. J Clin Oncol. 2008;26(15):2442-9.

39. Pao W, Miller V, Zakowski M, Doherty J, Politi K, Sarkaria I, et al. EGF receptor gene mutations are common in lung cancers from "never smokers" and are associated with sensitivity of tumors to gefitinib and erlotinib. Proc Natl Acad Sci U S A. 2004;101(36): 13306-11.

40. Inoue A, Suzuki T, Fukuhara T, Maemondo M, Kimura Y, Morikawa N, et al. Prospective phase II study of gefitinib for chemotherapy-naive patients with advanced non-small-cell lung cancer with epidermal growth factor receptor gene mutations. J Clin Oncol. 2006;24(21):3340-6.

41. Ding L, Getz G, Wheeler DA, Mardis ER, McLellan MD, Cibulskis $\mathrm{K}$, et al. Somatic mutations affect key pathways in lung adenocarcinoma. Nature. 2008;455(7216):1069-75.

42. Bai H, Mao L, Wang HS, Zhao J, Yang L, An TT, et al. Epidermal growth factor receptor mutations in plasma DNA samples predict tumor response in Chinese patients with stages IIIB to IV nonsmall-cell lung cancer. J Clin Oncol. 2009;27(16):2653-9.

43. Sos ML, Michel K, Zander T, Weiss J, Frommolt P, Peifer M, et al. Predicting drug susceptibility of non-small cell lung cancers based on genetic lesions. J Clin Invest. 2009;119(6):1727-40.

44. Harari PM, Wheeler DL, Grandis JR. Molecular target approaches in head and neck cancer: epidermal growth factor receptor and beyond. Semin Radiat Oncol. 2009;19(1):63-8.

45. Rubin Grandis J, Melhem MF, Gooding WE, Day R, Holst VA, Wagener MM, et al. Levels of TGF-alpha and EGFR protein in head and neck squamous cell carcinoma and patient survival. J Natl Cancer Inst. 1998;90(11):824-32.

46. Conde E, Angulo B, Tang M, Morente M, Torres-Lanzas J, LopezEncuentra A, et al. Molecular context of the EGFR mutations: evidence for the activation of mTOR/S6K signaling. Clin Cancer Res. 2006;12(3 Pt 1):710-7.

47. Kalyankrishna S, Grandis JR. Epidermal growth factor receptor biology in head and neck cancer. J Clin Oncol. 2006;24(17): 2666-72.

48. Sok JC, Coppelli FM, Thomas SM, Lango MN, Xi S, Hunt JL, et al. Mutant epidermal growth factor receptor (EGFRvIII) contributes to head and neck cancer growth and resistance to EGFR targeting. Clin Cancer Res. 2006;12(17):5064-73.

49. Temam S, Kawaguchi H, El-Naggar AK, Jelinek J, Tang H, Liu $\mathrm{DD}$, et al. Epidermal growth factor receptor copy number alterations correlate with poor clinical outcome in patients with head and neck squamous cancer. J Clin Oncol. 2007;25(16):2164-70.

50. Ang KK, Berkey BA, Tu X, Zhang HZ, Katz R, Hammond EH, et al. Impact of epidermal growth factor receptor expression on 
survival and pattern of relapse in patients with advanced head and neck carcinoma. Cancer Res. 2002;62(24):7350-6.

51. Chung CH, Ely K, McGavran L, Varella-Garcia M, Parker J, Parker $\mathrm{N}$, et al. Increased epidermal growth factor receptor gene copy number is associated with poor prognosis in head and neck squamous cell carcinomas. J Clin Oncol. 2006;24(25):4170-6.

52. Gupta AK, McKenna WG, Weber CN, Feldman MD, Goldsmith JD, Mick R, et al. Local recurrence in head and neck cancer: relationship to radiation resistance and signal transduction. Clin Cancer Res. 2002;8(3):885-92.

53. Kumar B, Cordell KG, Lee JS, Worden FP, Prince ME, Tran HH, et al. EGFR, p16, HPV Titer, Bcl-xL and p53, sex, and smoking as indicators of response to therapy and survival in oropharyngeal cancer. J Clin Oncol. 2008;26(19):3128-37.

54. O'Shea JJ, Gadina M, Schreiber RD. Cytokine signaling in 2002: new surprises in the Jak/Stat pathway. Cell. 2002;109(Suppl): S121-31.

55. Grandis JR, Drenning SD, Zeng Q, Watkins SC, Melhem MF, Endo $\mathrm{S}$, et al. Constitutive activation of Stat 3 signaling abrogates apoptosis in squamous cell carcinogenesis in vivo. Proc Natl Acad Sci U S A. 2000;97(8):4227-32.

56. Grandis JR, Drenning SD, Chakraborty A, Zhou MY, Zeng Q, Pitt AS, et al. Requirement of Stat3 but not Statl activation for epidermal growth factor receptor-mediated cell growth in vitro. J Clin Invest. 1998;102(7):1385-92.

57. Leeman RJ, Lui VW, Grandis JR. STAT3 as a therapeutic target in head and neck cancer. Expert Opin Biol Ther. 2006;6(3):231-41.

58. Sriuranpong V, Park JI, Amornphimoltham P, Patel V, Nelkin BD, Gutkind JS. Epidermal growth factor receptor-independent constitutive activation of STAT3 in head and neck squamous cell carcinoma is mediated by the autocrine/paracrine stimulation of the interleukin 6/gp130 cytokine system. Cancer Res. 2003;63(11): 2948-56.

59. Leeman-Neill RJ, Seethala RR, Singh SV, Freilino ML, Bednash JS, Thomas SM, et al. Inhibition of EGFR-STAT3 signaling with erlotinib prevents carcinogenesis in a chemically-induced mouse model of oral squamous cell carcinoma. Cancer Prev Res (Phila). 2011;4(2):230-7.

60. Cohen RB. Current challenges and clinical investigations of epidermal growth factor receptor (EGFR)- and ErbB family-targeted agents in the treatment of head and neck squamous cell carcinoma (HNSCC). Cancer Treat Rev. 2014;40(4):567-77.

61. Rosenthal EL, Chung TK, Carroll WR, Clemons L, Desmond R, Nabell L. Assessment of erlotinib as adjuvant chemoprevention in high-risk head and neck cancer patients. Ann Surg Oncol. 2014;21(13):4263-9. An important clinical study assessing the tolerability and efficacy of long-term erlotinib treatment in terciary chemoprevention in HNSCC/OSCC following salvage surgery.

62. Shin DM, Zhang H, Saba NF, Chen AY, Nannapaneni S, Amin ARMR, et al. Chemoprevention of head and neck cancer by simultaneous blocking of epidermal growth factor receptor and cyclooxygenase-2 signaling pathways: preclinical and clinical studies. Clin Cancer Res. 2013;19(5):1244-56.

63. Mestre JR, Chan G, Zhang F, Yang EK, Sacks PG, Boyle JO, et al. Inhibition of cyclooxygenase-2 expression. An approach to preventing head and neck cancer. Ann N Y Acad Sci. 1999;889: 62-71.

64. Lin DT, Subbaramaiah K, Shah JP, Dannenberg AJ, Boyle JO. Cyclooxygenase-2: a novel molecular target for the prevention and treatment of head and neck cancer. Head Neck. 2002;24(8): 792-9.

65. Saba NF, Hurwitz SJ, Kono SA, Yang CS, Zhao Y, Chen Z, et al. Chemoprevention of head and neck cancer with celecoxib and erlotinib: results of a phase $\mathrm{Ib}$ and pharmacokinetic study. Cancer Prev Res (Phila). 2014;7(3):283-91. A promising clinical study reporting on the effective chemopreventive action of the combined treatment with inhibitors of the EGFR (erlotinib) and COX-2 (celecoxib) pathways in a phase I and pharmacokinetic study in patients with advanced premalignant lesions.

66. Bailey CJ. Metformin: a multitasking medication. Diab Vasc Dis Res. 2008;5(3):156.

67. Evans JM, Donnelly LA, Emslie-Smith AM, Alessi DR, Morris $\mathrm{AD}$. Metformin and reduced risk of cancer in diabetic patients. BMJ. 2005;330(7503):1304-5

68. Pollak MN. Investigating metformin for cancer prevention and treatment: the end of the beginning. Cancer Discov. 2012;2(9): 778-90.

69. Pollak M. Potential applications for biguanides in oncology. J Clin Invest. 2013;123(9):3693-700.

70. Larsson O, Morita M, Topisirovic I, Alain T, Blouin MJ, Pollak M, et al. Distinct perturbation of the translatome by the antidiabetic drug metformin. Proc Natl Acad Sci U S A. 2012;109(23):897782

71. El-Mir MY, Nogueira V, Fontaine E, Averet N, Rigoulet M, Leverve X. Dimethylbiguanide inhibits cell respiration via an indirect effect targeted on the respiratory chain complex I. J Biol Chem. 2000;275(1):223-8.

72. Zhou G, Myers R, Li Y, Chen Y, Shen X, Fenyk-Melody J, et al. Role of AMP-activated protein kinase in mechanism of metformin action. J Clin Invest. 2001;108(8):1167-74.

73. Shaw RJ, Lamia KA, Vasquez D, Koo SH, Bardeesy N, Depinho RA, et al. The kinase LKB1 mediates glucose homeostasis in liver and therapeutic effects of metformin. Science. 2005;310(5754): $1642-6$.

74. Stephenne X, Foretz M, Taleux N, van der Zon GC, Sokal E, Hue L, et al. Metformin activates AMP-activated protein kinase in primary human hepatocytes by decreasing cellular energy status. Diabetologia. 2011;54(12):3101-10.

75. Vitale-Cross L, Molinolo AA, Martin D, Younis RH, Maruyama T, Patel V, et al. Metformin prevents the development of oral squamous cell carcinomas from carcinogen-induced premalignant lesions. Cancer Prev Res (Phila). 2012;5(4):562-73. This preclinical study reported for the first time on the benefits of the antidiabtic drug metformin in the chemoprevention of HNSCC/OSCC in a carcinogen-induced oral carcinogenesis mouse model.

76. Patel H, Younis RH, Ord RA, Basile JR, Schneider A. Differential expression of organic cation transporter OCT-3 in oral premalignant and malignant lesions: potential implications in the antineoplastic effects of metformin. J Oral Pathol Med. 2013;42(3):250-6.

77. Shrotriya S, Tyagi A, Deep G, Orlicky DJ, Wisell J, Wang X-J, et al. Grape seed extract and resveratrol prevent 4-nitroquinoline 1-oxide induced oral tumorigenesis in mice by modulating AMPK activation and associated biological responses. Mol Carcinog. 2013.

78. Warner BM, Casto BC, Knobloch TJ, Accurso BT, Weghorst CM. Chemoprevention of oral cancer by topical application of black raspberries on high at-risk mucosa. Oral Surg Oral Med Oral Pathol Oral Radiol. 2014;118(6):674-83.

79. Casto BC, Knobloch TJ, Galioto RL, Yu Z, Accurso BT, Warner BM. Chemoprevention of oral cancer by lyophilized strawberries. Anticancer Res. 2013;33(11):4757-66.

80. Wang W, Yang Y, Zhang W, Wu W. Association of tea consumption and the risk of oral cancer: a meta-analysis. Oral Oncol. 2014;50(4): 276-81.

81. Fu J-Y, Gao J, Zhang Z-Y, Zheng J-W, Luo J-F, Zhong L-P, et al. Tea consumption and the risk of oral cancer incidence: a casecontrol study from China. Oral Oncol. 2013;49(9):918-22.

82. Lipinski CA, Lombardo F, Dominy BW, Feeney PJ. Experimental and computational approaches to estimate solubility and permeability in drug discovery and development settings. Adv Drug Deliv Rev. 2001;46(1-3):3-26. 
83. Katiyar SK, Afaq F, Perez A, Mukhtar H. Green tea polyphenol (-)epigallocatechin-3-gallate treatment of human skin inhibits ultraviolet radiation-induced oxidative stress. Carcinogenesis. 2001;22(2): 287-94.

84. Surh YJ, Chun KS. Cancer chemopreventive effects of curcumin. Adv Exp Med Biol. 2007;595:149-72.

85. Goel A, Kunnumakkara AB, Aggarwal BB. Curcumin as "Curecumin": from kitchen to clinic. Biochem Pharmacol. 2008;75(4):787-809.

86. Azmi AS, Bhat SH, Hadi SM. Resveratrol-Cu(II) induced DNA breakage in human peripheral lymphocytes: implications for anticancer properties. FEBS Lett. 2005;579(14):3131-5.

87. Goswami SK, Das DK. Resveratrol and chemoprevention. Cancer Lett. 2009;284(1):1-6.

88. Xia EQ, Deng GF, Guo YJ, Li HB. Biological activities of polyphenols from grapes. Int J Mol Sci. 2010;11(2):622-46.

89. Si HY, Li DP, Wang TM, Zhang HL, Ren FY, Xu ZG, et al. Improving the anti-tumor effect of genistein with a biocompatible superparamagnetic drug delivery system. J Nanosci Nanotechnol. 2010;10(4):2325-31.

90. Ganguly A, Yang H, Cabral F. Paclitaxel-dependent cell lines reveal a novel drug activity. Mol Cancer Ther. 2010;9(11):2914-23.

91. Onyuksel H, Jeon E, Rubinstein I. Nanomicellar paclitaxel increases cytotoxicity of multidrug resistant breast cancer cells. Cancer Lett. 2009;274(2):327-30.

92. Sulfikkarali N, Krishnakumar N, Manoharan S, Nirmal RM. Chemopreventive efficacy of naringenin-loaded nanoparticles in 7,12-dimethylbenz(a)anthracene induced experimental oral carcinogenesis. Pathol Oncol Res POR. 2013;19(2):287-96.

93. Felgines C, Texier O, Morand C, Manach C, Scalbert A, Regerat F, et al. Bioavailability of the flavanone naringenin and its glycosides in rats. Am J Physiol Gastrointest Liver Physiol. 2000;279(6): G1148-54.

94. Mulshine JL, Ondrey FG. Not significant but important. Cancer Prev Res (Phila). 2013;6(5):371-4. 